\title{
Fluoroquinolone and macrolide co-resistance in clinical isolates of Campylobacter species: a 15-year study in Karachi, Pakistan
}

S. Irfan, ${ }^{7}$ A. Ahmad, ${ }^{7}$ D. Guhar, ${ }^{1}$ E. Khan, ${ }^{7}$ F. Malik, ${ }^{1}$ S. Mahmood' and A. Zafar ${ }^{7}$

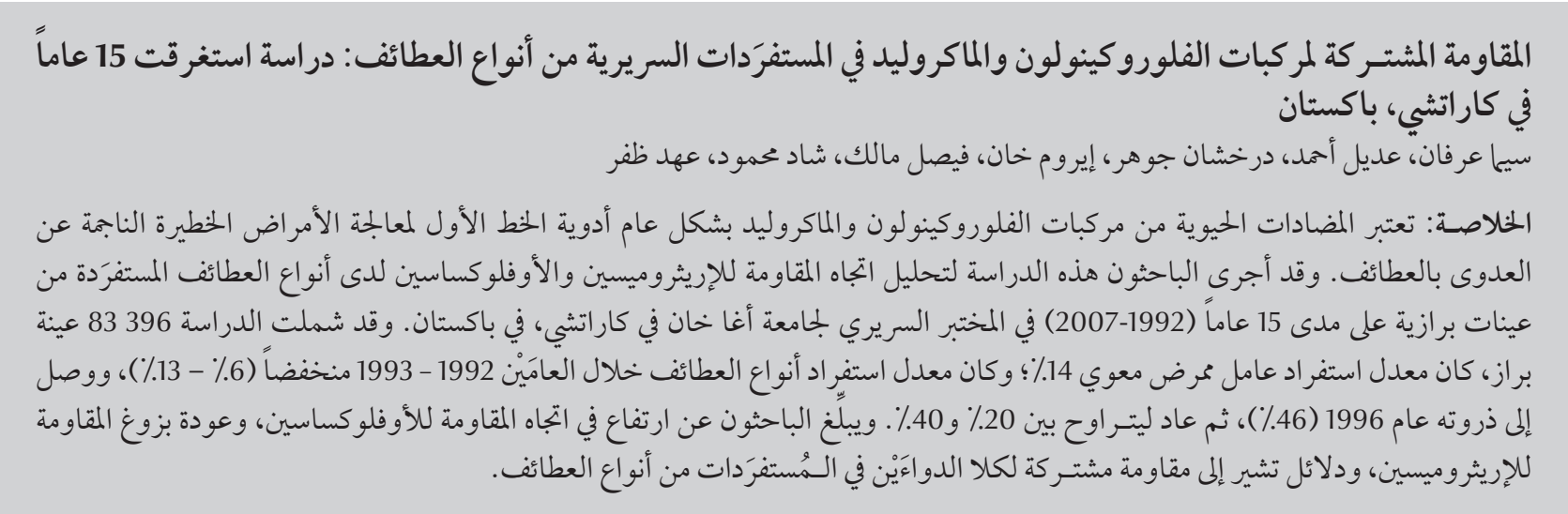

ABSTRACT Fluoroquinolone and macrolide antibiotics are generally considered as first-line drugs for the treatment of severe campylobacteriosis. This study was conducted to analyse the trend of erythromycin and ofloxacin resistance among Campylobacter spp. isolated from stool specimens over a period of 15 years (19922007) at The Aga Khan University clinical laboratory in Karachi, Pakistan. A total of 83396 stool specimens were processed, with a $14 \%$ isolation rate for enteric pathogens. The isolation rate of Campylobacter spp. was low during 1992-93 (6\%-13\%), peaked in 1996 (46\%), then ranged from 20\% to 40\%. We report a rising trend in ofloxacin resistance, re-emergence of erythromycin resistance and indications of co-resistance to both drugs in clinical isolates of Campylobacter spp.

Co-résistance aux fluoroquinolones et aux macrolides des isolats cliniques de Campylobacter : une étude sur 15 ans à Karachi (Pakistan)

RÉSUMÉ Les antibiotiques tels que les fluoroquinolones et les macrolides sont généralement indiqués dans le traitement de première ligne des cas sévères de campylobactériose. La présente étude a été conduite pour analyser la tendance de la résistance à l'érythromycine et à l'ofloxacine des isolats des espèces du genre Campylobacter dans des échantillons de selles recueillis sur une période de quinze ans (de 1992 à 2007) au laboratoire clinique de I'Université Aga Khan à Karachi (Pakistan). Au total, 83396 échantillons de selles ont été analysés, avec un taux d'isolement de $14 \%$ pour les agents pathogènes intestinaux. Le taux d'isolement de l'espèce Campylobacter est resté faible entre 1992 et 1993 (de 6 \% à 13\%), a atteint un pic en 1996 (46\%), puis s'est stabilisé de $20 \%$ à $40 \%$. Nous avons observé une tendance à l'augmentation de la résistance à l'ofloxacine, une réémergence de la résistance à l'érythromycine et des indications de co-résistance des isolats cliniques de Campylobacter aux deux substances. 


\section{Introduction}

Campylobacter spp. are the leading cause of enteritis in both developing countries and the developed world [1-3]. While a majority of Campylobacter infections are mild self-limiting diarrhoeal illnesses, in a proportion of cases severe infections occur, including prolonged enteritis, septicaemia and other extraintestinal infections [4]. Milder cases of campylobacteriosis do not require antimicrobial drugs but serious infections need therapy. In addition, immunocompromised travellers might require prophylaxis and treatment for enteritis [5]. Erythromycin and fluoroquinolone are generally considered as first- and second-line drugs for the treatment of Campylobacter infections.

Unfortunately antimicrobial resistance against these 2 groups of drug continues to rise throughout the world, especially against fluoroquinolone, resistance to which is rising at an alarming rate [6]. Fluoroquinoloneor macrolide-resistant Campylobacter strains are associated with increased risk of death and invasive disease as well as longer durations of illness in the immunocompetent population [7-9]. Data about the antimicrobial resistance of Campylobacter spp. is sparse in the developing world, especially from Pakistan. A study from The Aga Khan University reported Campylobacter spp. as the third most common pathogen in stool specimens [10].

The present study was conducted to analyse the rates of isolation of Campylobacter spp. isolated from stool specimens and the trend of fluoroquinolone and macrolide resistance over a period of 15 years at a referral laboratory in Karachi, Pakistan.

\section{Methods}

A retrospective study of stool culture datafrom January 1992 to June 2007 was conducted at the clinical microbiology laboratory of the Aga Khan University hospital, a tertiary referral centre based in Karachi, Pakistan. The laboratory receives specimens from both inpatients and outpatients from all over the country via its satellite collection centres.

The American Society of Microbiology (ASM) guidelines were used for the culture of stool samples throughout the study period [11]. For the isolation of Campylobacter spp., stool samples were inoculated onto agar-based Campylobacter medium, supplemented with $40 \%$ haemolysed sheep blood with added antibiotics: trimethoprim, polymyxin B and vancomycin. Plates were incubated for 48 hours in a microaerophilic environment at $42^{\circ} \mathrm{C}$ for selective isolation of Campylobacter spp. Seagull-shaped Gram-negative rods with the ability to produce oxidase and catalase enzymes were identified as Campylobacter spp. and further tested for hippurate hydrolysis and cephalothin susceptibility.

All isolated strains of Campylobacter spp. were tested for antimicrobial susceptibility by the Kirby-Bauer disk diffusion method against 5 drugs: erythromycin (a macrolide), ofloxacin (a fluoroquinolone), ampicillin, gentamicin and tetracycline. The zone diameter breakpoints of each antimicrobial agent were determined according to the Clinical and Laboratory Standards Institute established guidelines for bacteria isolated from animals $[12-15]$. The same procedure for Campylobacter spp. isolation and antimicrobial susceptibility testing was followed throughout the study period. Campylobacter jejuni ATCC 33291 was used as a quality control strain for identification and susceptibility testing.

\section{Results}

During the 15-year study period (January 1992-June 2007) a total of 83396 stool samples were processed, with a $14 \%$ isolation rate for enteric pathogens $(n=11653)$. Vibrio cholerae was the most frequently isolated (32\%) over this period, followed by Campylobacter spp. (30\%). The isolation rates of Salmonella spp. and Shigella spp. were $18 \%$ and $15 \%$ respectively.

Figure 1 shows the annual isolation rate of Campylobacter spp. from 1992 to 2007. The rate of isolation of Campylobacter spp. was low (6.3\%) in 1993, peaked in 1996 (46\%) and then for the next 10 years ranged from around $20 \%$ to over $40 \%$.

C. jejuni (89.5\%) was the main species isolated, followed by C. coli (6.4\%), C. laridis (3.2\%) and other Campylobacter spp. $(0.8 \%)$

The age distribution showed that the majority of the Campylobacter spp. isolates over the 15-year period (70\%) came from children aged $<5$ years (Figure 2).

The annual antimicrobial susceptibility data showed that ofloxacin resistance first emerged in 1994, rising to $25.4 \%$ in 2000 and continued to rise to reach a peak of $47.5 \%$ by 2004 (Figure 3). Ofloxacin resistance was highest in C. jejuni (21\%), followed by C. coli (9\%). Erythromycin resistance was $9.5 \%$ in 1993 , gradually decreasing to $0 \%$ in 2000 but re-emerged in 2001 and gradually rose to reach $2.9 \%$ in 2005 (Figure 3). Erythromycin resistance was highest in C. coli (5\%), followed by C. jejuni (3\%). Co-resistance to both erythromycin and ofloxacin was $0.5 \%$. The overall rates of resistance against ampicillin, tetracycline and gentamicin were $22 \%, 13 \%$ and $4 \%$ respectively.

\section{Discussion}

This study reviewed the isolation rate and trend of antimicrobial resistance of Campylobacter spp. in Karachi, Pakistan over a period of 15 years. To the best of our knowledge this is the longest period of reporting from this region. The isolation rate of Campylobacter spp. was lowest during the first 2 years, peaked in 1996 and since then has fluctuated 


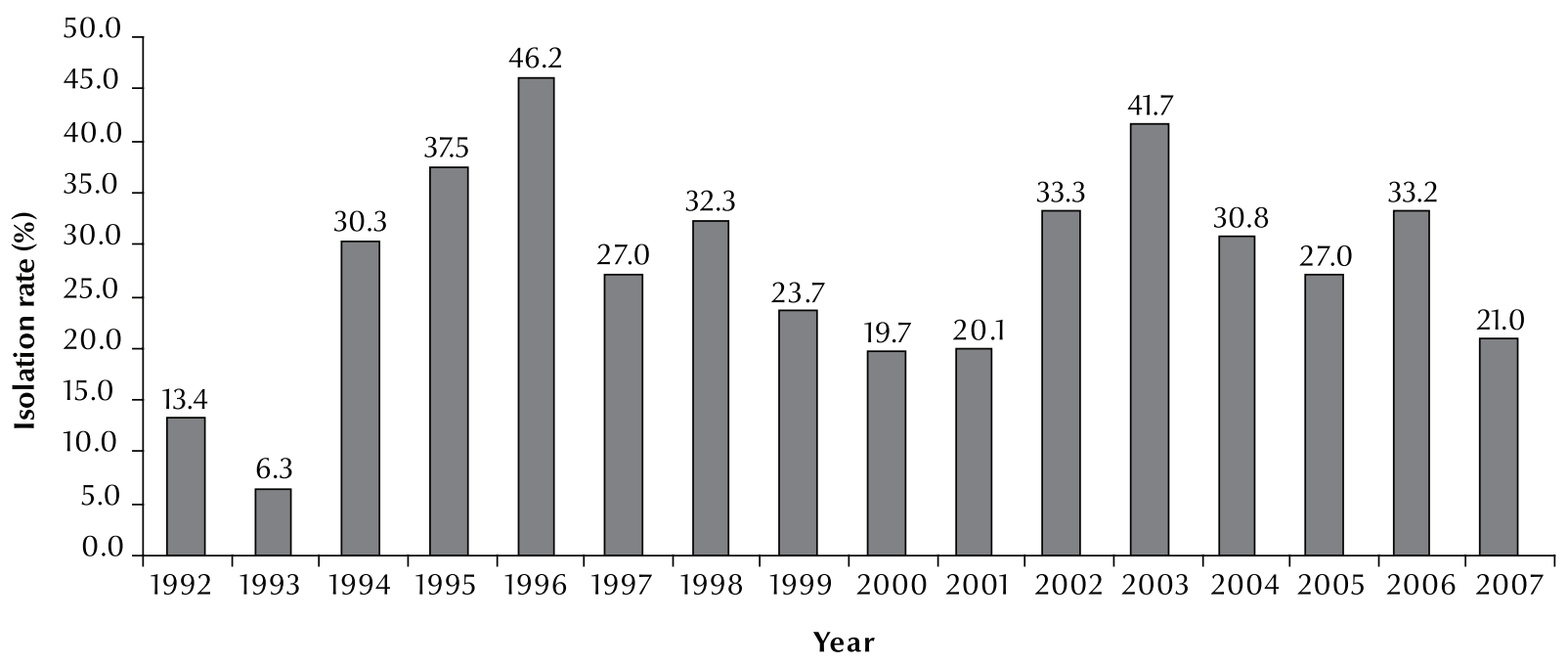

Figure 1 Annual Campylobacter spp. isolation rate from stool samples at a referral hospital in Karachi, January $1992-J u n e ~ 2007$

between $20 \%$ and $40 \%$. A study from this centre has previously reported this organism to be the third most commonly isolated organism (24\%) after V. cholerae (31\%) and Salmonella spp. (26\%). However, current data showed a very similar isolation rate of this organism as for $V$. cholerae over the study period, i.e. $30 \%$ versus $32 \%$. The reason for the change in this epidemiology is not clear. A study published from Bangladesh also found Campylobacter spp. with the highest frequency among stool pathogens [16]. C. jejuni was the most common species found (89.5\%), as reported in other studies [17].

In our study, the rate of isolation of Campylobacter spp. was highest among children aged $<5$ years, which is consist-

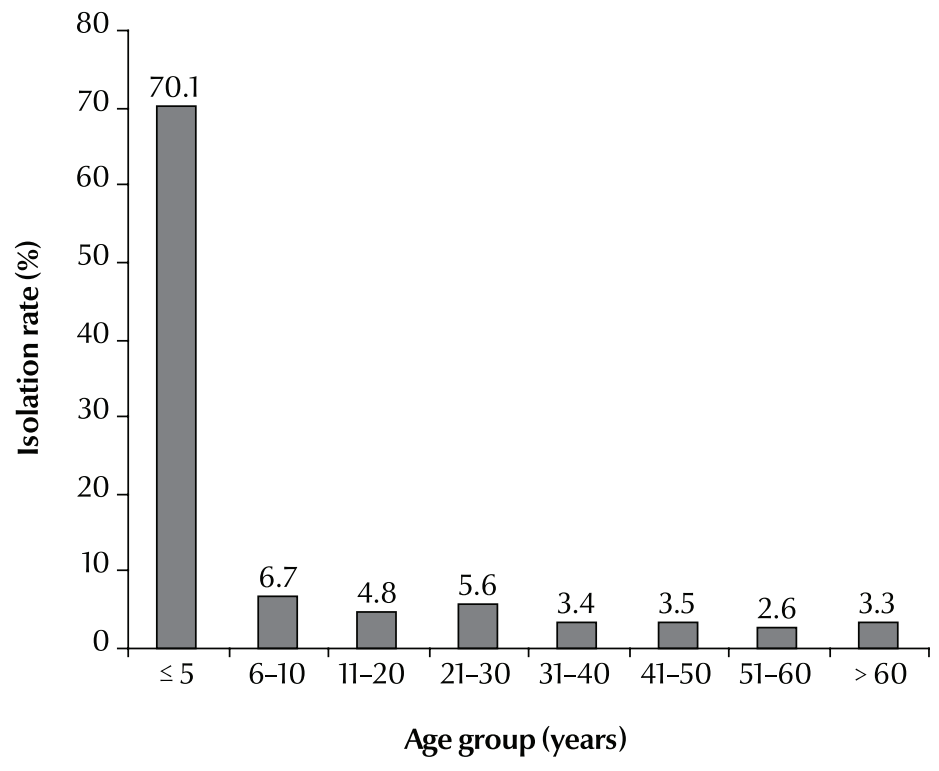

Figure 2 Isolation of Campylobacter spp. in different age groups from stool samples at a referral hospital in Karachi, January 1992-June $2007(n=3384)$ ent with a previous study reported from this region [18].

Our findings showed a gradual rise of fluoroquinolone resistance from its emergence in 1994 to $25.4 \%$ in 2000 and $47.5 \%$ in the year 2004 . The rising trend is comparable with other studies published from different parts of the world $[19,20]$. Fluoroquinolones are among the most commonly used agents to treat Campylobacter enteritis in the community. However, since the early 1990s resistance against this group of drugs has been reported from several countries [20-22]. The prophylactic use of fluoroquinolones in veterinary medicine as well as their use as a growth promoter in animal husbandry was recognized as a cause of resistance in the developed world [23]. A study published from Australia reported a significant fall in fluoroquinolone resistance after restricting its use in foodproducing animals [24]. However, in a developing country such as Pakistan, the major issue is injudicious use of antibiotics in humans especially when there is a high rate of self-medication.

Another important finding of this study is the re-emergence of macrolide resistance. The literatureshows thatresistance to macrolides is already high in Far East countries such as Singapore (51\%), 


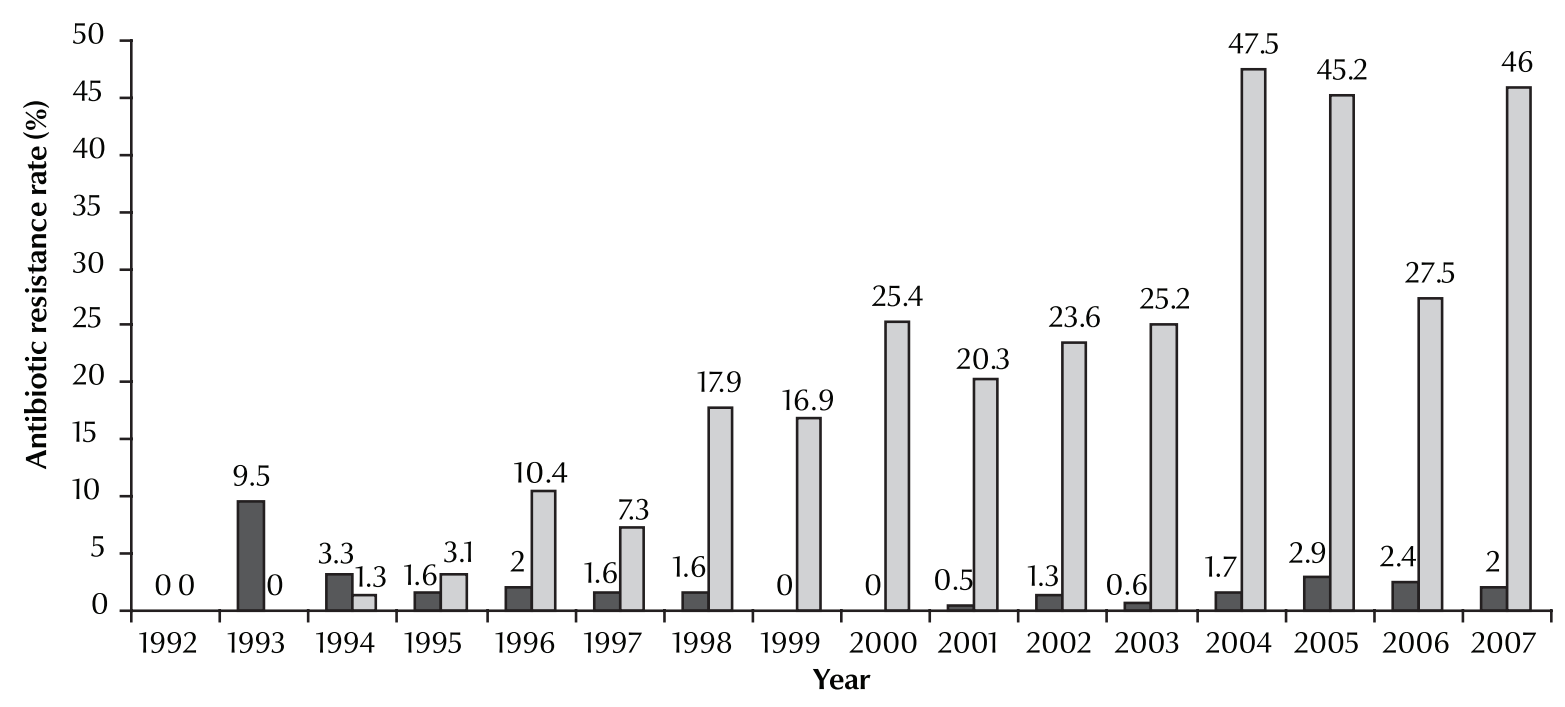

Figure 3 Annual rate of resistance to erythromycin and ofloxacin of Campylobacter spp. isolated from stool samples at a referral hospital in Karachi, January 1992-June 2007

Thailand (31\%) and Taiwan (18\%) [25]. However, our rates are closer to the rates of developed countries [26].

An important finding of our study was the demonstration of co-resistance to the macrolide and fluoroquinolone agents tested by a few Campylobacter spp. isolates $0.5 \%$ ). The emerging coresistance must be considered alarming as these 2 classes are generally considered as first- and second-line agents. Moreover, most of these isolates showed resistance to other groups of antibiotics, thus limiting the choice for treatment of life-threatening infections. Recent studies have compared the clinical symptoms, duration of illness and mortality rates between fluoroquinolone-/macrolide-sensitive and resistant isolates and concluded that drug-resistant strains had a significant relationship to disease severity and mortality [27].

As our study was laboratory-based, the clinical outcome of these cases could not be evaluated. However, the study highlights the need for future clinical studies based on clinical outcome, along with genetic analysis and epidemiological typing of these multidrug resistant Campylobacter spp. isolates.

\section{Conclusion}

In view of the minimum resistance of Campylobacter spp. against the macrolide group of antibiotics, they can be continued as empirical therapy for severe campylobacteriosis in both the paediatric and adult population in Karachi, Pakistan. However, the empirical use of fluoroquinolones is debatable due to rising resistance of Campylobacter spp. to this group of antibiotics.

\section{Acknowledgements}

This study was supported through a grant from the Joint Pakistan-US Academic and Research Program HEC/ MoST/USAID.

We would like to thank the faculty and staff of the clinical microbiology laboratory of the Aga Khan University hospital, Karachi for their support and help in this study.

\section{References}

1. Allos BM 2001: Campylobacter jejuni infections: update on emerging issues and trends. Clinical Infectious Diseases, 32:1201-1206.

2. Friedman CR et al. Epidemiology of Campylobacterjejuni infections in the United States and other industrialized Nations. In: Nachamkin I, Blaser MJ, eds. Campylobacter. Washington DC, ASM Press, 2000:121-139.

3. Miller M et al. Australia's notifiable disease status 2003: annual report or the National Notifiable Diseases Surveillance System. Communicable Diseases Intelligence, 2005, 29:1-61.
4. Helms M et al. Short and long term mortality associated with foodborne bacterial gastrointestinal infections: registry based study. British Medical Journal, 2003, 326:357 (doi:10.1136/ bmj.326.7385.357).

5. Norrby SR: Principles of chemoprophylaxis. In: Finch RG, et al., eds. Antibiotic and chemotherapy, 8th ed. New York, Elsevier Science, 2003:120-122.

6. Nachamkin I, Ung H, Ming I. Increasing fluoroquinolone resistance in Campylobacter jejuni, Pennsylvania, USA, 1982-2001. Emerging Infectious Diseases, 2002, 8:1501-1503. 
7. Nelson JM et al. Prolonged diarrhea due to ciprofloxacinresistant Campylobacter infection. Journal of Infectious Diseases, 2004, 190:1150-1177.

8. Engberg J. Quinolone-resistant Campylobacter infections: risk factors and clinical consequences. Emerging Infectious Dis eases, 2004, 10:1056-1063.

9. McCellan $\mathrm{J}$ et al and NARMS working group. Prevalence and consequences of floroquinolone-resistant Campylobacter infection: NARMS 1997-2000 (slide session 48). In: Proceedings of the International Conference on Emerging Infectious Diseases Atlanta, Georgia, February 29 to March 3, 2004. (http://www. cdc.gov/narms, accessed 4 May 2010).

10. Ibrahim NG, Zafar A, Hasan R. Evaluation of frequency of isolation and trends in antibiotic resistance among Campylobacter isolates over 11-year period. Journal of the Pakistan Medical As sociation, 2004, 54:291-294.

11. York MK et al. Fecal and other gastrointestinal cultures and toxin assays. In: Isenberg HD, ed. Clinical microbiology procedures handbook. Washington DC, American Society for Microbiology Press, 2004:3.8.1.1-3.8.2.19

12. Centers for Disease Control and Prevention. National antimicrobial resistance monitoring system: enteric bacteria, 2001 annual report. Atlanta, Georgia, National Antimicrobial Resistance Monitoring System, 2003.

13. Andrews JM. BSAC standardized disc susceptibility testing method (version 6). Journal of Antimicrobial Chemotherapy, 2007, 60:20-41.

14. National Committee for Clinical Laboratory Standards. Performance standards for antimicrobial disk and dilution susceptibility tests for bacteria isolated from animals approved standard M31-A2. Wayne, Pennsylvania, National Committee for Clinical Laboratory Standards, 2002

15. National Committee for Clinical Laboratory Standards. Performance standards for antimicrobial susceptibility testing, 12th informational supplement M100-S72. Wayne, Pennsylvania, National Committee for Clinical Laboratory Standards, 2002.

16. Hoque SS et al. Infectious agents causing acute watery diarrhoea in infants and young children in Bangladesh and their public health implications. Journal of Tropical Pediatrics, 1994, 40(6):351-354.

17. Sack DA, Lyke C, McLaughlin C. Antimicrobial resistance in shigellosis, cholera and campylobacteriosis. World Health Organization, Department of Communicable Disease Surveillance and Response, 2001 (HO/CDS/CSR/DRS/2001.8).

18. Ali AM et al. Antibiotic resistance in Campylobacter jejuni in Rawalpindi and Islamabad-a preliminary study. Pakistan Journal of Medical Sciences, 2003, 19:272-276.

19. Luber $\mathrm{P}$ et al Antimicrobial resistance in Campylobacter jejuni and Campylobacter coli strains isolated in 1991 and 2001-2002 from poultry and humans in Berlin, Germany. Antimicrobial Agents and Chemotherapy, 2003, 47:3825-3830.

20. Gaudreau C, Giblert H. Antimicrobial resistance of Campylobacter jejuni subspecies Jejuni strains isolated from humans in 1998 to 2001 in Montreal, Canada. Antimicrobial Agents and Chemotherapy, 2003, 47:2027-2029.

21. Iovine N, Blaser MJ. Antibiotics in animal feed and spread of resistant Campylobacter from poultry to humans. Emerging Infectious Diseases, 2004, 10:1158-1159.

22. Gaudreau C, Michaud S. Cluster of erythromycin and ciprofloxacin-resistant Campylobacter jejuni subspecies from 1999 to 2001 in men who have sex with men, Quebec, Canada. Clinical Infectious Diseases, 2003, 37:131-136.

23. Unicomb LE et al. Low level fluoroquinolone resistance among Campylobacter jejuni isolates in Australia. Clinical Infectious Diseases, 2006, 42:1368-1374.

24. Cooper DC. Emerging mechanisms of floroquinolone resistance. Emerging Infectious Diseases, 2001, 2:337-341.

25. Hees $\mathrm{V}$ et al. Regional and seasonal differences in incidence and antibiotic resistance of Campylobacter from a nationwide surveillance study in the Netherlands: an overview of 20002004. Clinical Microbiology and Infection, 2007, 13:305-310.

26. Engberg J et al. Quinolone and macrolide resistance in Campylobacter jejuni and C. coli: resistance mechanisms and trends in human isolates. Emerging Infectious Diseases, 2001, 1:24-33.

27. Mølbak K. Human health consequences of antimicrobial drugresistant Salmonella and other foodborne pathogens. Clinical Infectious Diseases, 2005, 41:1613-1620. 